CARNETS DE Carnets de géographes

GÉOGRAPHES.

$1 \mid 2010$

Varia

\title{
Le carnet de voyage : approches historique et sémiologique
}

\section{Pascale Argod}

\section{(2) OpenEdition}

Journals

Édition électronique

URL : http://journals.openedition.org/cdg/1995

DOI : $10.4000 /$ cdg. 1995

ISSN : 2107-7266

Éditeur

UMR 245 - CESSMA

Référence électronique

Pascale Argod, «Le carnet de voyage : approches historique et sémiologique », Carnets de géographes [En ligne], 1 | 2010, mis en ligne le 01 octobre 2010, consulté le 24 septembre 2020. URL : http:// journals.openedition.org/cdg/1995; DOI : https://doi.org/10.4000/cdg.1995

Ce document a été généré automatiquement le 24 septembre 2020.

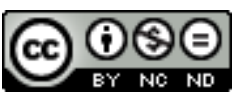

La revue Carnets de géographes est mise à disposition selon les termes de la Licence Creative Commons Attribution - Pas d'Utilisation Commerciale - Pas de Modification 4.0 International. 


\title{
Le carnet de voyage : approches historique et sémiologique
}

\author{
Pascale Argod
}

1 Le carnet de voyage, récemment apparu dans l'édition, depuis environ vingt ans, ne fait l'objet d'un engouement que depuis une dizaine d'année. Ce genre éditorial et artistique mineur a été souvent déprécié et assimilé au brouillon, voire au palimpseste, de l'artiste peintre. L'évolution rapide, en dix ans, de ce médium reflèterait en effet les problématiques actuelles des sciences de l'information et de la communication liées à diverses mutations. Du livre au numérique, de l'image fixe au flux d'images protéiformes, de l'objet hybride à l'intermédialité, de la singularité du médium à sa combinaison multimédia, de l'œuvre picturale à l'œuvre mosaïque et à l'art métis, de l'œuvre d'art vers la diffusion de masse, le carnet de voyage s'inscrirait dans la diffusion médiatique et migrerait vers le reportage télévisuel. De l'art aux mass médias, le carnet de voyage tendrait à devenir un produit culturel et marketing, reflet sociologique de lecteurs devenus spectateurs, de voyageurs de l'imaginaire devenus voyeurs de l'ailleurs, de curieux du monde devenus consommateurs de médias, d'artistes devenus surtout des communicants.

2 La thèse porte sur un essai de définition du genre « carnet de voyage » comme album hybride à la croisée du documentaire et du livre d'artiste à partir d'un panorama de l'édition (800 titres en corpus) et d'une recherche historique des emprunts, des croisements et des interactions en arts et en sciences humaines qui seraient à l'origine du mélange des genres. Les objectifs de la recherche sont essentiellement de caractériser le carnet de voyage et de proposer des typologies, du carnet de recherches au livre d'artiste ou encore au guide touristique. Entre reportage graphique et métissage artistique, vision exotique et ethnographie, il ferait l'objet d'une hybridation, d'une circulation artistique et d'une intermédialité, enjeux sémiologiques du carnet de voyage contemporain qui semblent propices à des développements communicatifs et éducatifs autour de la notion d'interculturel et de médiation de l'altérité. La thèse se construit donc selon un déroulement chronologique en trois parties, de la genèse du genre à sa création dans l'édition puis à ses déclinaisons actuelles, accompagné 
d'études d'exemples destinés à cerner le genre, à en circonscrire ses limites mais aussi les marges qui définissent le processus d'hybridation de l'album. À partir de l'image fixe artistique graphique, définie comme esquisse ou illustration, différentes notions sont abordées afin de qualifier l'image dialectique de « reportage graphique » selon deux aspects: l'objectivité du documentaire et la subjectivité du livre d'artiste. La définition contemporaine du carnet de voyage devient complexe car elle met en exergue deux notions "l'intermédialité »-transposition et circulation médiatiqueavec l'artialité et "l'interculturel»-apport culturel, métissage culturel, médiation transculturelle- qui en font un objet de communication attractif, notamment pour la formation et l'enseignement.

Il m'a semblé opportun de dresser, dans une première partie, une recherche historique aux origines de l'ethnographie et aux sources de l'œuvre picturale à travers l'esquisse. Le carnet de voyage est lié à l'histoire de l'ethnographie et donc de la discipline de l'ethnologie- anthropologie mais aussi au voyage d'exploration (XVIe siècle) ou de formation artistique et humaniste (XVIIe siècle). Dépendant de l'histoire du livre d'artiste et de l'esquisse de l'œuvre picturale, l'héritage est marqué par des peintres illustres dont certains ont une vision ethnographique de l'ailleurs (Delacroix et Gauguin). J'ai essayé de circonscrire le sujet par d'autres approches afin de situer le genre dans une vision globale, historique et culturelle, de l'image fixe de type "illustration de l'ailleurs » et de définir certains enjeux historiques, techniques et culturels qui permettent d'appréhender l'engouement ou le désintérêt, fluctuant au fil de l'histoire, pour le genre lié au voyage d'exploration, à l'art pictural et aux arts graphiques. Diffusé à grande échelle, il devient un produit visuel et culturel qui obéit aux lois du marché. La tendance actuelle du carnet de voyage répondrait en effet à des besoins et des critères sociologiques et de marketing. Aussi l'édition contemporaine se situe-t-elle entre guide touristique et livre d'art et obéit au développement du tourisme et des destinations appréciées qui permettent à l'édition de vendre, enjeu d'un marketing ciblé et contraignant.

4 Au croisement des arts et de la géographie, le carnet de voyage pose la problématique de l'intention de l'auteur artiste et reporter: du reportage graphique entre création artistique et réalisation documentaire; s'agit-il d'émouvoir, de raconter ou d'informer? Cet "album autobiographique de voyage" semble au cœur d'une vulgarisation par l'image de la connaissance d'une culture et pose la question du statut du «reportage graphique». Définir le «reportage graphique contemporain» m’a semblé déterminant car il semblerait que, lorsque la limite entre le reportage et l'autobiographie s'estompe, il s'agisse plutôt d'un "carnet de voyage» que d'un " reportage graphique ». La création plastique repose sur cette subjectivité sensorielle et émotionnelle que le carnet de voyage place au centre de son propos. De plus, la vision sur l'ailleurs est-elle bornée à une dimension culturelle ou est-elle interculturelle et métissée de deux cultures (celle de l'auteur et celle de l'objet)? Mon propos a donc porté sur les rapports entre l'anthropologie du voyage, l'ethnologie, l'ethnographie, la sémiologie de l'image et les visions du monde proposées à travers ce médium, exposé à la Biennale du carnet de voyage de Clermont-Ferrand du mois de novembre.

Fiche informative 
Discipline

Sciences de l'information et de la communication

\section{Directeur}

M. Lancier

\section{Université}

Université Michel de Montaigne-Bordeaux 3, équipe de recherche GRESIC-MICA (EA 44 26)

Membres du jury de thèse, soutenue le 10 décembre 2009

- M. Gilles Delavaud, Professeur à l'université de Paris VIII

- M. Rémi Hess, Professeur à l'université de Paris VIII

- Mme Martine Joly, Professeur à l'université de Bordeaux III

- M. Lancien, Professeur à l'université de Bordeaux III

- M. Philippe Viallon, Professeur à l'université de Genève

\section{Situation professionnelle actuelle}

PRCE à l'Université Montesquieu de Bordeaux IV, équipe de recherche GRESICMICA ((EA 44 26) de l'Université Michel de Montaigne-Bordeaux III, filière « Médias » sous la direction de M. Pierre Beylot http://mica.u-bordeaux3.fr/ et http://gresic.u-bordeaux3.fr/

\section{Contact de l'auteur}

0557870939 / 0678944649

pascale.argod[at]aquitaine.iufm.fr

\section{INDEX}

Thèmes : Carnets de soutenances 